\title{
Universality and RG Explanations
}

\author{
Robert W. Batterman* \\ Department of Philosophy \\ University of Pittsburgh
}

August 17, 2017

\section{Contents}

1 Introduction 1

2 Universality of Critical Phenomena:

A Paradigm Case 2

3 The Ingredients of Universality 4

3.1 Order Parameters and Symmetries _... . . . . . . . . . 4

3.2 Length Scales . . . . . . . . . . . . . . . . . . . 6

4 How Universality is Defined 8

4.1 Stability . . . . . . . . . . . . . 10

5 Explaining Universality 12

6 Objections and Responses 16

6.1 Objections: Reutlinger and Lange . . . . . . . . . . . . . 17

6.2 Objections: Franklin and Mainwoood . . . . . . . . . . 18

$\begin{array}{lll}7 \text { Conclusion } & 21\end{array}$

*Thanks to Michael Miller and Porter Williams for helpful comments and discussions. 


\section{Introduction}

In its broadest sense, "universality" is a technical term for something quite ordinary. It refers to the existence of patterns of behavior by physical systems that recur and repeat despite the fact that in some sense the situations in which these patterns recur and repeat are different. Rainbows, for example, always exhibit the same pattern of spacings and intensities of their bows despite the fact that the rain showers are different on each occasion. They are different because the shapes of the drops, and their sizes can vary quite widely due to differences in temperature, wind direction, etc. There are different questions one might ask about such patterns. For instance, one might ask why the particular rainbow that I'm currently seeing exhibits the spacings and intensities of its bows that it does. Perhaps an answer to that question might need to refer to the particular sizes and shapes of the drops in the particular rain shower at this time. On the other hand, one might ask about how it is possible that despite the differences in the lower scale details about the sizes and shapes of drops in different rain showers, the spacings and intensities of the bows in the different rainbows are the same. This latter question concerns the explanation of the (universal) pattern of behavior. It is arguable that the answer to the former question (or even answers to the former question for all the different rainbows all taken together) cannot answer the second question. [Batterman(2002)]

This paper examines what is, in the physics literature, the paradigm example of universality; namely the so-called universality of critical phenomenacertain kinds of phase transitions that systems (fluids and magnets, e.g.) can undergo. In the next section I describe the phenomena. In section 3 I lay out the mathematical and physical ingredients required for one to describe this universal pattern. These include the introduction of a function called an "order parameter" that serves to represent a surprising change of symmetry as a system passes through a so-called critical point. Section 4 looks back to some early work on phase transitions to see how the concept of universality should be properly defined. One of the most important features is a kind of stability of macroscopic behavior under changes of microscopic details. Any explanation of the possibility of universal behavior must account for this type of stability. In section 5 I briefly discuss how the renormalization group (RG) can explain the existence of universal behavior, in part by explaining the existence of this kind of stability. Finally, in section 6 I address some objections to the RG explanation of universality that have been raised repeatedly in the 
philosophical literature.

\section{Universality of Critical Phenomena: A Paradigm Case}

One of the most striking examples of universal behavior concerns the pattern displayed by molecularly distinct fluids near their so-called critical points. It is worth spending a bit of time examining this paradigmatic example of universality.

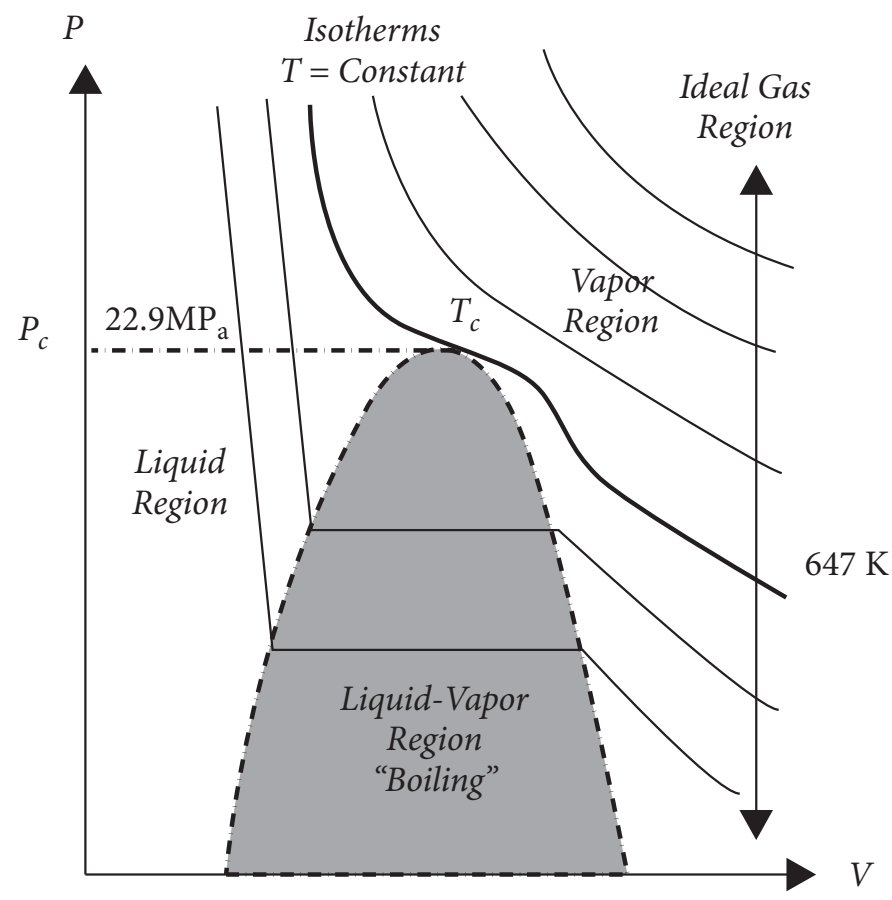

Figure 1: Cartoon PVT Diagram for Water [Kadanoff(2013), p. 148]

Everyone knows that water can exist in three distinct phases: as a liquid, as a solid, and as vapor or gas. One can represent these different phases graphically using the thermodynamic variables, pressure $(P)$, volume $(V)$, and temperature $(T)$. In figure 1, the curves show how the pressure depends on the volume at different fixed values for the temperature. Consider the 
boiling region. This corresponds to the process that takes place when water boils in a kettle. Inside the kettle both vapor and liquid coexist. The special point $\left(T_{c}, P_{c}\right)$ is called the critical point. It is special in the following way. Below the critical temperature $\left(T_{c}=647 \mathrm{~K}\right)$ and critical pressure $\left(P_{c}=22.9 \mathrm{MPa}\right)$ one finds the region of liquid/vapor coexistence. Above that critical point, the kettle will no longer contain two distinct phases of water. There is an abrupt change in the makeup of the stuff in the kettle at that critical temperature and pressure. For carbon dioxide (as for many other fluids) the diagram looks exactly the same although there will be different values for the critical temperature and pressure. ${ }^{1}$ In saying that the diagram looks exactly the same for carbon dioxide as it does for water, the important thing is that the shapes of the dotted lines near $\left(T_{c}, P_{c}\right)$ for both fluids are identical. So, while water and carbon dioxide are very different fluids as is evidenced by their very different critical temperatures and pressures, nevertheless near their critical points ("near criticality") they exhibit identical behavior. This is universal behavior realized by molecularly very different fluids.

A remarkable representation of the experimental fact of universality is provided by a figure in E. A. Guggenheim's 1945 paper entitled "The Principle of Corresponding States." When plotted in reduced coordinates $\left(\frac{\rho}{\rho_{c}}, \frac{T}{T_{c}}\right)$, the coexistence curves for eight different fluids near criticality all collapse onto the same curve. See figure 2.

One can quantify the universal behavior by introducing a so-called "order parameter." For the transition between the boiling region with two coexistent phases and the region above, define the order parameter $\Psi$ to be the difference between the densities of the liquid and the vapor in the kettle:

$$
\Psi=\left|\rho_{l}-\rho_{v}\right|
$$

Then the relation ${ }^{2}$

$$
\Psi \propto \epsilon^{\beta}
$$

describes the shape of the coexistence curve for a fluid. Universality is also expressed by the fact that $\beta$ is the same for the different fluids. This is just what is represented in figure 2 .

\footnotetext{
${ }^{1}$ For carbon dioxide, $T_{c}=31.1 C(=304.3 \mathrm{~K})$ and $P_{c}=7.2 \mathrm{MPa}$. Experimentally, it is a lot easier to realize the critical temperature and pressure of $\mathrm{CO}_{2}$ than it is for water.

${ }^{2} \epsilon=\left|\left(T_{c}-T\right) / T\right|$ and is a measure of how close a system is to its critical temperature in dimensionless units.
} 


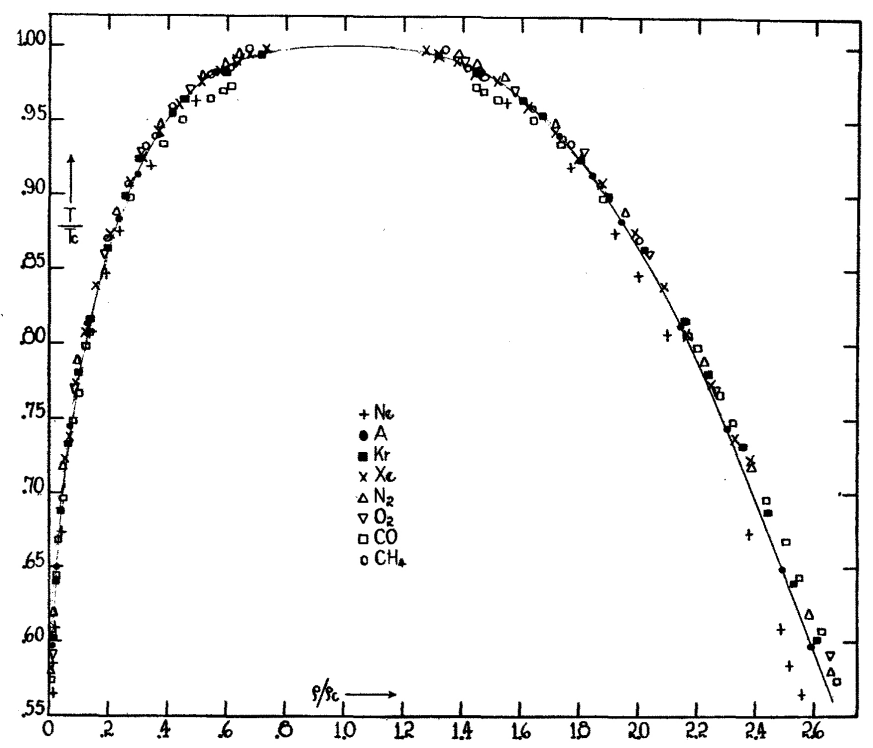

Figure 2: Universality of Critical Phenomena [Guggenheim(1945)]

Guggenheim's demonstration of the data collapse is indeed striking. But it is even more remarkable that the critical behavior of magnets exhibits the identical scaling relation. For a ferromagnet the order parameter is $M$, the net magnetization, and the relation

$$
M \propto \epsilon^{\beta}
$$

holds as well with $\beta$ identical to the value in equation (1).

\section{The Ingredients of Universality}

\subsection{Order Parameters and Symmetries}

Exactly what kind of quantities are the order parameters $\Psi$ and $M$ ? One can think of them as thermodynamic properties that allow us to characterize the qualitative behavior of systems near their critical points. That is, one can treat these as thermodynamic properties on a par with pressure, temperature, and volume. As thermodynamic properties they describe the behavior to be expected as a system is cooled from a temperature above $T_{c}$ to below $T_{c}$. In the case of $\Psi$ we see that as that temperature is crossed, there is a 
spontaneous appearance of two new states of matter (liquid and vapor). In the case of $M$, the magnet exhibits zero net magnetization (it is in a socalled paramagnetic phase) and spontaneously gains a net magnetization as the critical temperature is crossed. In both cases, above $T_{c}$ there exists a symmetry that is broken upon passing through $T_{c}$. For example, in a magnet in zero external magnetic field, there is rotational symmetry (no preferred direction) above $T_{c}$ that is broken upon passing through $T_{c}$ : All of a sudden there is a preferred direction of magnetization.

The concept of an order parameter was first introduced ${ }^{3}$ by Landau in 1937. [Landau(1965), pp. 193-216] The order parameter, as noted, captures the macro or continuum behavior and reflects the symmetry changes in a fluid as a parameter (temperature) is varied. But, from the point of view of statistical mechanics, one needs to think about $\Psi$ and $M$ in a different way. What, after all, is responsible for there being non-zero values of $M$ below $T_{c}$ ? The answer has to depend on some kind of lower scale/microscopic features of the magnet - some fact about the arrangement of (magnetic) spins on a lattice. Michael Fisher puts this as follows:

To assert that there exists an order parameter in essence says: "I may not understand the microscopic phenomena at all" (as was historically, the case for superfluid helium), "but I recognize that there is a microscopic level and I believe it should have certain general, overall properties as regards locality and symmetry: those then serve to govern the most characteristic behavior on scales greater than atomic." [Fisher(1998), p. 654]

Once one sees the order parameter as coding for some feature of the $m i$ crostructure of the magnet or fluid, one is in the domain of statistical physics. Now one needs to treat the order parameters as averages and one needs to consider the possibility of fluctuations in the values of the order parameters. Furthermore, thinking like this actually requires that one distinguish between the macroscopic scale (the scale of the continuum where the order parameter is simply a function of thermodynamic macroscopic properties like temperature and pressure), a mesoscale where fluctuations in aggregates of atomic scale properties may be important, and the atomic scale where what matters is the detailed natures of the atoms/molecules and spins. The order parameter lives in the intermediate region. Here is Fisher again:

\footnotetext{
${ }^{3}$ Michael Fisher [Fisher(1998), p. 654] says it's fair to say Landau invented the order parameter.
} 
Significantly, in my view, Landau's introduction of the order parameter exposed a novel and unexpected foliation or level in our understanding of the physical world. Traditionally, one characterizes statistical mechanics as directly linking the microscopic world of nuclei and atoms (on length scales of $10^{-13}$ to $10^{-8} \mathrm{~cm}$ ) to the macroscopic world of say, millimeters to meters. But the order parameter, as a dynamic, fluctuating object in many cases intervenes on an intermediate or mesoscopic level characterized by scales of tens or hundreds of angstroms up to microns (say, $10^{-6.5}$ to $\left.10^{-3.5} \mathrm{~cm}\right)$. [Fisher(1998), pp. 654]

It is reasonable to ask why this works. Why is this foliation of the physical world successful and appropriate? The answer to this is important for understanding both how universal behavior is possible and for understanding how one can explain such universality. That is to say, the existence of mesoscale features of the world captured by the order parameter is a necessary condition for universality. And, the explanation of universal behavior employs methods that exploit the fact that there is a wide separation of scales between the atomic and the macroscopic with no characteristic lengths in between.

\subsection{Length Scales}

Kadanoff describes a "very interesting and fundamental question" concerning the fact that the world

shows an amazing variety of length scales: There is the Hubble radius of the universe, $10^{10}$ light years or so and the radius of our own solar system, $10^{11}$ meters roughly, and us-two meters perhaps, and an atom $-10^{-10}$ meters in radius, and a proton $10^{-16}$ meters, and the characteristic length of quantum gravity — which involves another factor of about $10^{20}$.

How these vastly different lengths arise is a very interesting and fundamental question. ... However, we can think about how one describes the region between these lengths. If one is looking at scales between two fundamental lengths, there are no nearby characteristic lengths. Similarly in critical phenomena, whenever one looks [at] any event which takes place between the scale 
of the lattice constant [the spacing between molecules or spins] and the much larger scale of the coherence length, one is in a situation in which there are no nearby characteristic lengths. [Kadanoff(2000), p. 251]

This lack of characteristic length scales is crucial for both the existence of universal behavior and for its explanation. In the above quote, Kadanoff refers to the "coherence length" sometimes also called the "correlation length." For a system near criticality the correlation length becomes enormous and for infinite systems, it diverges to infinity. ${ }^{4}$ For a fluid system like the water in the kettle, the correlation length is a measure of the average size of a region of vapor (say) in the kettle. Vapor molecules cluster with other vapor molecules and liquid molecules cluster with other liquid molecules. (The analog of this in a ferromagnet is that the spins on the lattice sites want to be next to spins pointing in the same direction. So one has "droplets" of up-spins of a certain size and "droplets" of down-spins as well.) As the water in the boiling region (or the spins in the magnet) heat up and approach critical temperatures, the size of the different droplets get larger and larger. This means that, even though the physical forces between the molecules (or spins) remain local, distant molecules (spins) become correlated as a result of existing in the different droplets. In addition, one has large correlated droplets of vapor inside droplets of liquid inside droplets of vapor .... See figure 3.

In the phase diagram of figure 1 , the region near critical point inside the boiling region corresponds to this "fractal like" structure of droplets within droplets, etc. The reason for is that near the critical point, fluctuations are dominant and average values for the order parameters essentially lose their meaning. Orthodox statistical mechanics is unable to describe the critical behavior because there are fluctuations at all length scales from the macroscopic correlation length equal to the size of the system (the kettle), and the microscopic distance of the range of the forces between molecules or spins. These fluctuations "cannot probe all the details of the interatomic potential. Rather they only see certain gross features of the potential: for example the amount of breaking of an exact symmetry ... or the distance from the critical point." [Kadanoff(1971), p. 104]

Given all these details about what is happening at the micro-, meso-, and macro-scales in the neighborhood of a critical point, we can now see how properly to define the universality of critical phenomena.

\footnotetext{
${ }^{4}$ I will have more to say about the role of infinite systems below.
} 


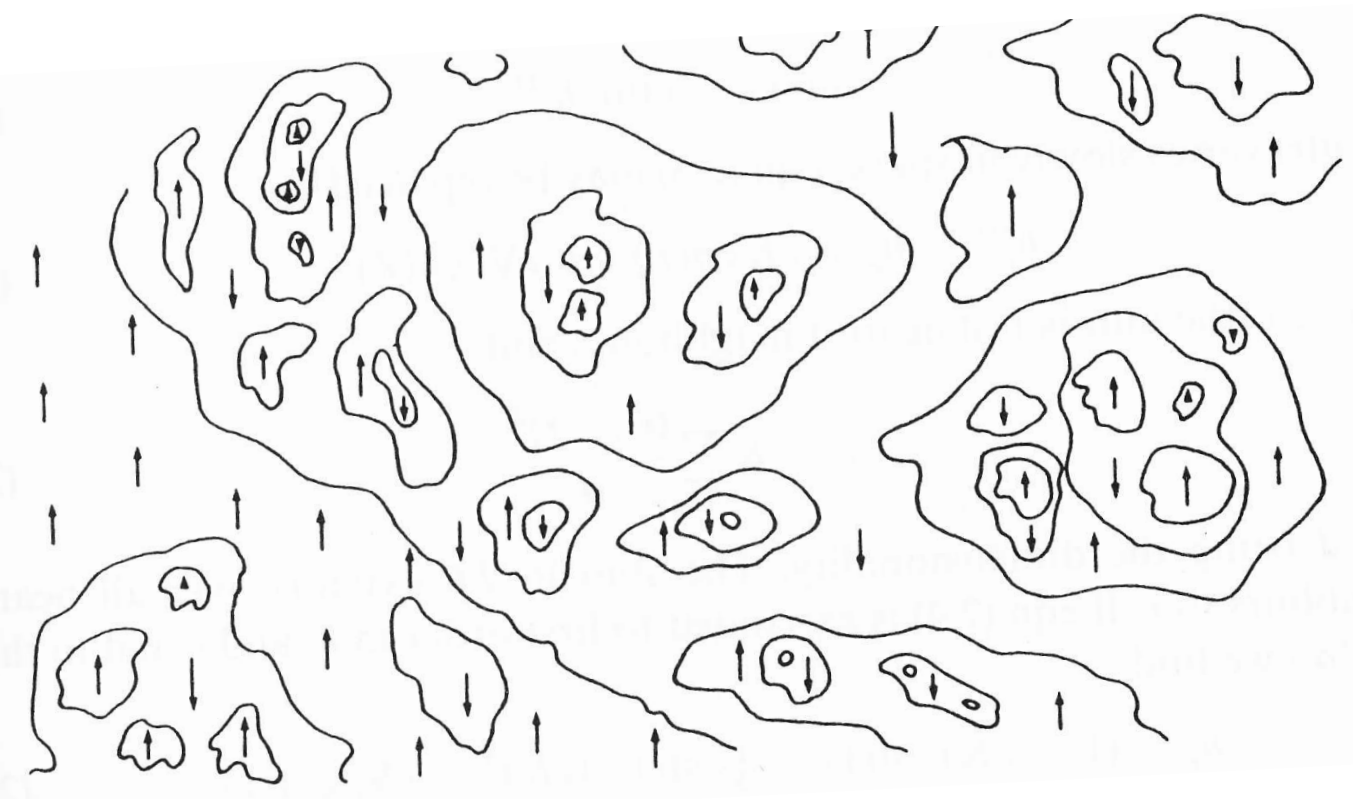

Figure 3: Droplets inside droplets inside droplets ... [Kadanoff(1976), p. 12]

\section{How Universality is Defined}

Kadanoff states the "hypothesis of universality" as follows:

All phase transition problems can be divided into a small number of different classes depending upon the dimensionality of the system and the symmetries of the order state. Within each class, all phase transitions have identical behaviour in the critical region, only the names of the variables are changed. [Kadanoff(1971), p. 103]

Table 1 exhibits different values for the scaling exponent $\beta$ for different phase transitions at criticality. ${ }^{5}$ We can here see how the scaling exponent depends upon the dimensionality of the system. The ferromagnetic transition for a two dimensional film is in a different universality class than the three dimensional magnet. Notice also that despite remarkable differences

\footnotetext{
${ }^{5}$ Table 1 is from data presented in [Kadanoff(1971), p. 102].
} 
in microstructural makeup, the liquid/vapor systems, superfluid helium, and the $d=3$ ferromagnet are all in the same universality class; that is, the scaling exponent $\beta$ appearing in equations (1) and (2) are the same (within experimental error).

Table 1: Scaling Exponents for Different Transitions

\begin{tabular}{l|c}
\hline Phase Transition & Value of $\beta$ \\
\hline Mean Field Theory & $1 / 2$ \\
$d=2$ Ising Model Ferromagnet & $1 / 8$ \\
$d=3$ Liquid/Vapor $\mathrm{CO}_{2}$, Xe & 0.35 \\
$d=3$ Superfluid Helium ${ }^{4} \mathrm{He}$ & 0.359 \\
$d=3$ Ising Model Ferromagnet & 0.315 \\
\hline
\end{tabular}

A full description of a phase transition typically involves two field variables. Consider the ferromagnetic transition. One can take the external magnetic field $h$ as a field that can drive a system from one coexisting phase (up spins) to the other (down spins). The second field is provided by the reduced temperature, $\epsilon$, defined above in footnote 2 . This field drives the system closer or away from the critical point. These are the variables to which Kadanoff refers in his hypothesis of universality above. In terms of these field variables, the free energy for the system can be written as follows ${ }^{6}$ : $F=F(h, \epsilon)$. In differential form, this becomes

$$
d F=<M>d h+<\mathcal{H}>d \epsilon
$$

where $\langle\mathcal{H}\rangle$ is an energy. In this differential form we see that the fields $h$ and $\epsilon$ are paired with two thermodynamically conjugate variables: $\langle M\rangle$ and $\langle\mathcal{H}\rangle$, respectively. For the liquid/vapor transition, the corresponding pairs of variables are the order parameter $\Psi$ with its conjugate $\left(\mu-\mu_{c}\right)$ related to the chemical potential. When Kadanoff says that "only the names of the variables change" for systems in the same universality class these are the changes to which he refers:

$$
(M, h) \leftrightarrow\left(\Psi,\left(\mu-\mu_{c}\right)\right)
$$

\footnotetext{
${ }^{6}$ The free energy is a measure of the internal energy of the system that is available to do work.
} 
The ferromagnetic/paramagnetic phase transition and the liquid/vapor phase transition are in the same relatively small class.

\subsection{Stability}

Thus there is a relationship between different phase transitions problems that leaves invariant various features of those transitions. Here is Kadanoff again:

The theorist can discuss this relation in the following way: He imagines that yet another field is inserted into the free energy. Call that other field $\lambda$ and the operator which is its thermodynamic conjugate, $U$. Here $\lambda$ represents a parameter in the Hamiltonian. Continuous variation from $\lambda=0$ to $\lambda=1$ might represent the change in the Hamiltonian which takes us from the Ising model to the Heisenberg model, or from $\mathrm{Ni}$ to $\mathrm{Fe}$ or from a nearest neighbor interaction to a next nearest neighbor interaction. Therefore, the discussion of $\lambda$ and its thermodynamic conjugate $U$ is in effect the discussion of the relationship among different phase transition problems. [Kadanoff(1971), p. 105]

Consider the ferromagnetic transition. Inserting this new parameter into the Hamiltonian means that the free energy is now a function, not only of the magnetic field $h$ and the field $\epsilon$, but also of the field $\lambda$ :

$$
F=F(\epsilon, h, \lambda) .
$$

In differential form we now have

$$
d F=<M>d h+<\mathcal{H}>d \epsilon+<U>d \lambda .
$$

As an example, consider the Hamiltonian for a nearest neighbor Ising ferromagnet:

$$
\mathscr{H}_{\Omega}=-J_{n . n .} \sum_{<i j>} \sigma_{i} \sigma_{j}-h \sum_{i \in \Omega} \sigma_{i},
$$

where $\Omega$ is a region of spins on a $d$-dimensional lattice, $\langle i j\rangle$ signals nearest neighbor pairs on the lattice, $J_{n . n}$. characterizes the nearest neighbor spinspin coupling, and $h$ is a (uniform) magnetic field. 
Next, let

$$
\lambda=\frac{J_{n . n . n .}}{J_{n . n .}}
$$

be the ratio of the next nearest neighbor spin coupling strength to that of nearest neighbor coupling strength. Consider the variation of from $\lambda=0$ to $\lambda=1 .^{7}$ This takes us from the nearest-neighbor to next-nearest-neighbor coupling. Let $\epsilon$ and $h$ be defined as follows:

$$
\epsilon=\frac{\left(T-T_{c}(\lambda)\right)}{T_{c}(\lambda)} \text { and } \quad h=\frac{\mu_{\beta} H_{z}}{K T_{c}} .
$$

For $\lambda=0$ write the order parameter as function, $m_{0}$ of $h$ and $\epsilon$ :

$$
<M>=m_{0}(h, \epsilon) .
$$

Similarly introducing the distance $r$ between spins, one can write the spinspin correlation function $g_{0}$ as follows:

$$
<\sigma_{z}(0) \sigma_{z}(r)>=g_{0}(h, \epsilon, r) .
$$

Then Kadanoff [Kadanoff(1971), p. 106] asserts that "[u]niversality implies for $\lambda \neq 0 "$ :

$$
<M>=a m_{0}(\bar{h}, \bar{\epsilon}), \quad<\sigma_{z}(0) \sigma_{z}(r)>=\left(a b / d^{3}\right) g_{0}(\bar{h}, \bar{\epsilon}, \bar{r}),
$$

where

$$
\bar{h}=b h, \quad \bar{\epsilon}=c \epsilon, \quad \bar{r}=d r .
$$

This means that the functional forms of $\langle M\rangle$ and $\left\langle\sigma_{z}(0) \sigma_{z}(r)\right\rangle$ do not change as $\lambda$ varies.

Universality, then

... implies that the basic thermodynamic functions and correlation functions only depend on $\lambda$ via a trivial change of variables. The functional form is the same as at $\lambda=0$. However, the variables in these functions are changed in that $h, \epsilon$, and $M$ are each multiplied by parameters $a, b, c, d$ which depend upon $\lambda$. [Kadanoff(1971), p. 105]

\footnotetext{
${ }^{7}$ See also the paper by Robert Griffiths "Dependence of Critical Indices on a Parameter." [Griffiths(1970)]
} 
What does this result mean? It means that the Hamiltonians of different systems - as different as ${ }^{8}$ nickel and iron, or as different $\mathrm{as}^{9} \mathrm{CO}_{2}$, Xe, and ${ }^{4} \mathrm{He}$ - can be perturbed into one another without changing the nature of the phase transition problem. ${ }^{10}$ This, in turn, means that the scaling behavior of the order parameter will remain unchanged as the transformation (by varying the value of $\lambda$ ) between Hamiltonians is effected. In other words, many of the details that genuinely distinguish a lattice of nickel from that of iron (different interatomic strengths, etc.) are irrelevant for the scaling behavior. This is best understood as a stability result. The class of systems represented by their Hamiltonians between which such $\lambda$-transformations can take place without effecting the scaling behavior of the order parameter (whether it is $M$, or $\Psi$, or whatever) is called a "universality class." It is defined as that set of systems between which such (perturbative) transformations hold.

This stability under perturbation is the key property of universality. To explain how universality is possible, then, requires that one explain two features:

1. Why are the phase transitions stable under perturbation of the microscopic details of the systems (as encoded in their Hamiltonians)?

2. Why are the universality classes dependent ${ }^{11}$ upon the symmetry of the order parameter and the dimensionality of the systems?

\section{$5 \quad$ Explaining Universality}

In this section I outline, very briefly, how the renormalization group allows for an explanation of the two key features of universal behavior just mentioned. This explanation has been called into question by a number of commentators and I will consider their objections in the following section. Recall the ingredients of universality. Near criticality, the correlation length is enormous and there is a droplets-within-droplets structure that exhibits self-similarity (i.e,

\footnotetext{
${ }^{8}$ See above quote from Kadanoff.

${ }^{9}$ See Table 1 above.

${ }^{10}$ Not all such transitions will preserve the nature of the phase transition problem. In fact, a perturbation from an Ising Hamiltonian to a Heisenberg Hamiltonian, will take us from one problem to another.

${ }^{11}$ Recall Kadanoff's "hypothesis of universality" quoted at the beginning of this section.
} 


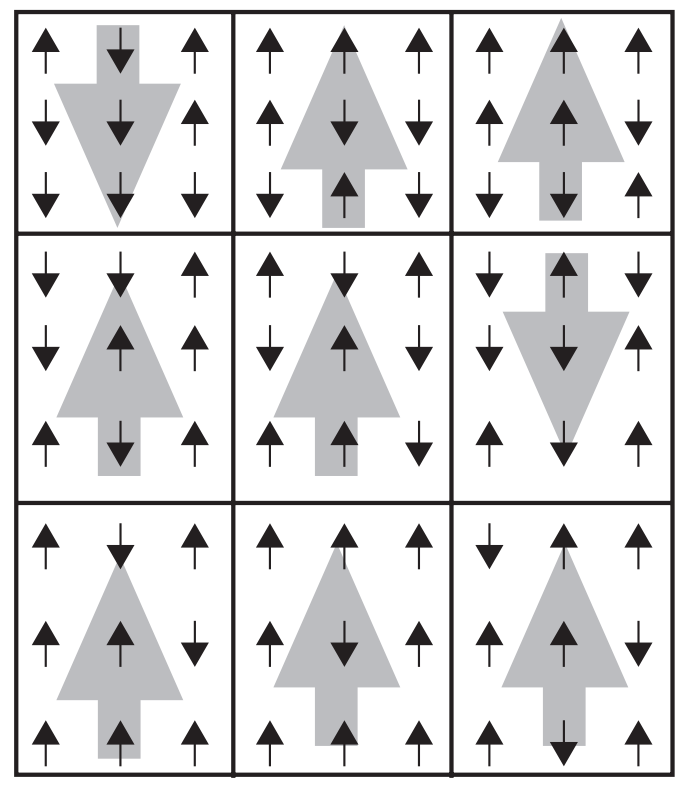

Figure 4: Blocking and averaging to yield a new (coarse-grained) effective system [Kadanoff(2013), p. 172]

it behaves like a fractal). As a result there are no characteristic scales between the atomic/lattice spacing and the continuum. Importantly, as noted, for an infinite system the correlation length actually diverges to infinity.

Kadanoff recognized that one could exploit the droplets-within-droplets fractal structure to change a Hamiltonian representing a system into a related effective Hamiltonian by a kind of coarse-graining procedure. This is now known as the Kadanoff block spin method.

The idea is to group or block spins or molecules and replace them with an some kind of average. ${ }^{12}$ In figure 4 there are nine spins per block and the "averaging" rule is to let the majority rule: If more spins in a block are up-spins (down-spins), replace those nine spins with a single "block spin" that points up (down). Now we have a lattice of block spins that looks pretty much like the original lattice but the spacings between the block spins is greater. Next one spatially rescales so as to put the new spins on the

\footnotetext{
${ }^{12}$ It almost doesn't matter what averaging or coarse-graining scheme is used. This fact is just another signature of the stability of phase transitions under perturbation.
} 


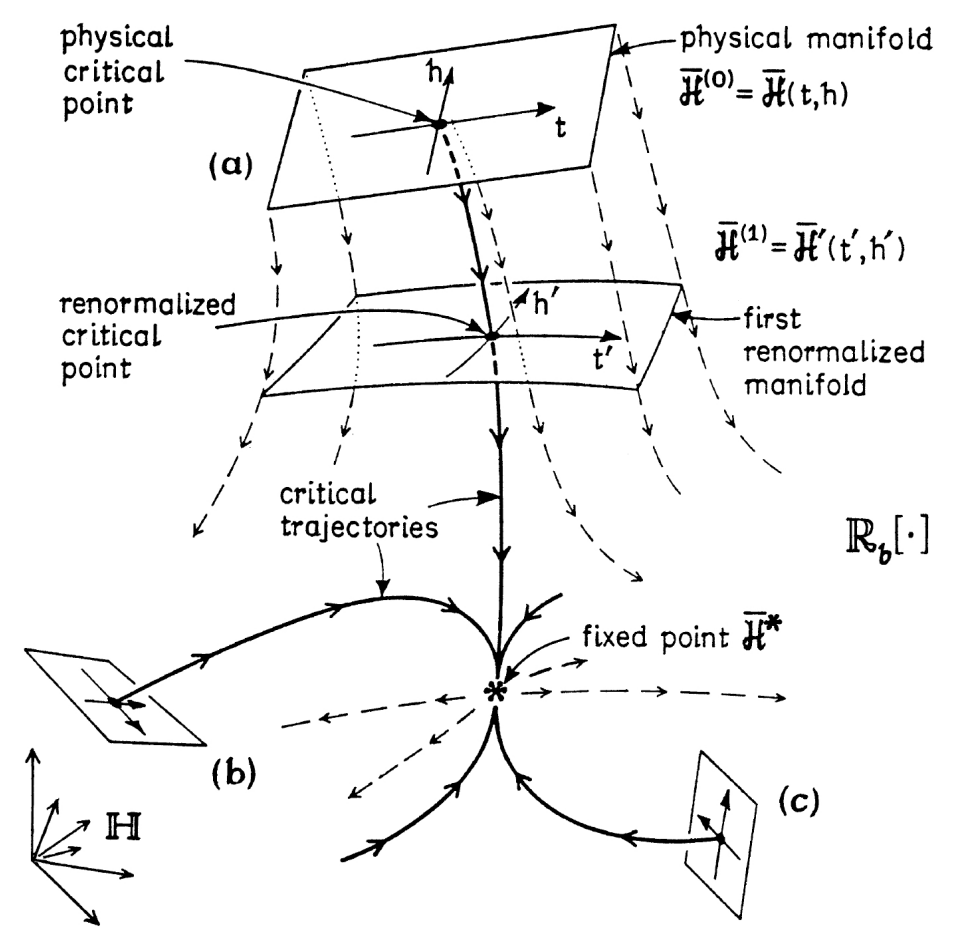

Figure 5: Fixed Point and Universality Class [Fisher (1998) 673]

same lattice as the original. Finally one changes the block spins so that the exhibit coupling strengths as similar to the original coupling as possible. ${ }^{13} \mathrm{We}$ now have a new "renormalized" Hamiltonian corresponding to this effective system. Continued iteration of this procedure leads to a flow (the RG flow) on an abstract space of Hamiltonians. That is, it induces a dynamics that takes one Hamiltonian into another as the blocking procedure is repeated. See figures 5 and 6.

One examines the dynamical flow on this abstract space and looks for potential fixed points. These are points which when acted upon by the transformation yield the same point. ${ }^{14}$ A fixed point is a property of the transformation itself and all details of the systems that flow toward that fixed point have been eliminated. Those systems/models (points in the space)

\footnotetext{
${ }^{13}$ This part of the procedure is to a certain extent an art. There is no explicit recipe that one can follow.

${ }^{14}$ If $\tau$ represents the transformation and $p^{*}$ is a fixed point we will have $\tau\left(p^{*}\right)=p^{*}$.
} 


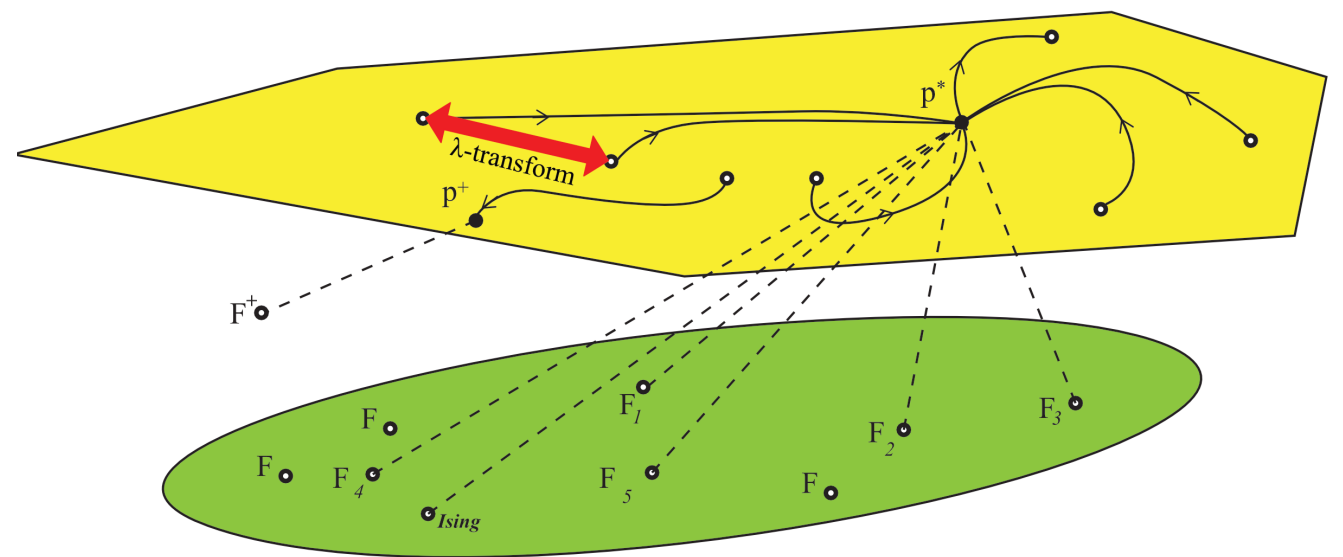

Figure 6: Fixed Point, Universality Class, and $\lambda$-Transformation

that flow to the same fixed point are in the same universality class - the universality class is delimited - and they will exhibit the same macro scaling behavior. ${ }^{15}$ That macro-behavior, in particular, the determination of the scaling exponent $\beta$ in equations (1) and (2) can be determined by an analysis of the transformation in the neighborhood of the fixed point.

Crucially, those systems that actually flow to a fixed point are at criticality. This means that they are infinite systems. As such, those systems are idealizations. But the infinite idealization is necessary if one is to locate the fixed points of the RG flow in the abstract space. This is because the correlation length must diverge to be able to infinitely iterate the RG transformation. Nevertheless, systems that are near criticality (real, large finite systems) will start off close to the critical systems and their behavior can be understood by examining the topology of the $\mathrm{RG}$ flow in the neighborhood of the fixed point. So, the RG explains the behavior of near critical, real systems. ${ }^{16}$ It explains what is going on in the neighborhood of the critical

\footnotetext{
${ }^{15}$ To put this another way: The universality class is the basin of attraction of the fixed point.

${ }^{16}$ If it only explained the behavior of idealized infinite systems, it would not be such a
} 
point in the boiling region of figure 1.

So the fixed point delimits the class of systems that all exhibit similar behavior near criticality. It also, thereby, justifies the existence of the kind of perturbative stability Kadanoff describes in his discussion of what we've called the $\lambda$-transformations. See, figure 6 . Finally, this analysis also demonstrates that the only important or relevant features (other than being near criticality) for this common behavior are the dimensionality of the system and the symmetry of the order parameter in the critical region.

\section{$6 \quad$ Objections and Responses}

As noted, a number of philosophers of science have challenged some of the claims I have just been making. Let me set up my replies to these objections with another quote from Michael Fisher. In "Scaling, Universality, and Renormalization Group Theory" [Fisher(1983)] Fisher expresses what, from a contemporary philosophical perspective, is a rather heretical point of view.

The traditional approach of theoreticians, going back to the foundation of quantum mechanics, is to run to Schrödinger's equation when confronted by a problem in atomic, molecular or solid state physics! One establishes the Hamiltonian, makes some (hopefully) sensible approximations and then proceeds to attempt to solve for the energy levels, eigenstates and so on. However, for truly complicated systems in what, these days, is much better called "condensed matter physics," this is a hopeless task; furthermore, in many ways it is not even a very sensible one!

The modern attitude is, rather, that the task of the theorist is to understand what is going on and to elucidate which are the crucial features of the problem. For instance, if it is asserted that the exponent $[\beta]$ depends on the dimensionality, $d$, and on the symmetry number, $n$, but on no other factors, then the theorist's job is to explain why this is so and subject to what provisos. If one had a large enough computer to solve Schrödinger's equation and the answer came out that way, one would still have no understanding of why this was the case!' [Fisher(1983), pp. 46-47]

big deal. Hardly worthy of a Nobel prize! 
It is clear from this quote that for Fisher our understanding of universal behavior requires explaining why the pattern depends upon the dimension of the system and the symmetry of the order parameter. This, of course, fits nicely with Kadanoff's definition of universality (section 4) that explicitly characterizes phase transition problems as depending upon the dimensionality of the system and the symmetries of the order state.

\subsection{Objections: Reutlinger and Lange}

Alexander Reutlinger holds that the RG explanation for the universal behavior of critical phenomena (section 5) proceeds in a completely different, intuitive, everyday way. He says RG explanations "are not special and quite intuitive in one crucial respect. RG explanations explain the phenomenon that microscopically different physical systems display the same macro-behavior ... by referring to features that those physical systems have in common, although the physical systems at issue are different in many other respects." [Reutlinger(2017), p. 144] The "common features" that are supposed to explain the universal behavior are the common dimensionality and the common symmetry of the order parameters. To cite these features, according to Ruetlinger, just is to explain the universal critical behavior of fluids and magnets.

In a reply to a paper by Collin Rice and me, Marc Lange [Lange(2015)] argues similarly for what Ruetlinger calls the "commonality strategy." He holds that our justification of why minimal models like the Ising model can be used to understand the behaviors of actual systems also depends upon citing common features. He asserts that "since the model's explanatory utility arises from its having certain features in common with the target system, the model's explanatory utility arises from its representing accurately enough the target system, contrary to B\&R's [Batterman's and Rice's] view." [Lange(2015), p. 298] Specifically, the claim is that a minimal or "toy" model can be explanatory if it shares features (dimension and symmetry in the case of the Ising model) with the actual target, fluid system.

In our paper, Rice and I argue that the justification for the applicability of minimal models derives from the demonstration that the minimal model is in the same universality class as the target system of interest. The discussion above aims in part to show how the RG demonstrates this very fact. Furthermore, and importantly, we have seen that this demonstration also provides an account of why dimensionality and symmetry are important 
features characteristic of the universality class.

But Lange asks why do " $\mathrm{B} \& \mathrm{R}$ insist that a minimal model explanation must explain why the common features are necessary for the macrobehavior to occur and why these features are present in all members of the universality class despite their heterogeneous microdetails?" [Lange(2015), p. 303] The answer to these questions, once one properly understands what it is to be a universality class, is that one needs to show that those very features (symmetry and dimensionality) are the important common features. This fits with the above discussion of the definition of universality (section 4) and with the insistence by Fisher (in the quote above) that "the theorist's job is to explain why [the common exponent $\beta$ depends upon the dimensionality $d$ and the symmetry number $n$ ]. [Fisher(1983), pp. 47]

Lange does appeal to a passage from Fisher that comes just after the one quoted above:

We may well try to simplify the nature of a model to the point where it represents a 'mere caricature' of reality. But notice that when one looks at a good political cartoon one can recognize the various characters even though the artist has portrayed them with but a few strokes. ... [A] good theoretical model of a complex system should be like a good caricature: it should emphasize those features which are most important and should downplay the inessential details." [Fisher(1983), p. 47]

Lange claims that here Fisher "seems to be supporting a 'common features account': the minimal model, despite being a caricature of some actual system, shares with it 'those features which are most important.' "[Lange(2015), p. 299, fn. 3]

Of course Lange is right about this. But the question is why are those the features that are important! Without an answer to that question, we neither

have a justification for the use of the minimal model, nor an explanation of universal behavior.

\subsection{Objections: Franklin and Mainwoood}

Alexander Franklin has recently argued that the Kadanoff blocking scheme (aka "real"-space RG) discussed above, cannot explain the possibility of universal behavior. [Franklin(2017)] He says that 
[b]uilding on Mainwood [Mainwood(2006)] I argue that the [realspace RG and the field-theoretic RG] approaches ought to be distinguished: while the field-theoretic approach explains universality, the real-space approach fails to provide an adequate explanation. [Franklin(2017), p.1]

Without going into too much detail, I want to argue that this claim is mistaken. The error is two-fold. First, the claim rests on a mistaken characterization of universality (similar to the view held by of Reutlinger and Lange). On this mistaken view, one fails to realize that to explain universality (as I've discussed above) requires that one explain the dependence of the macropattern on the symmetry and dimension. Second, Franklin's assertion rests upon a myth (whose genesis in philosophical discussions, I believe, is Mainwood's dissertation [Mainwood(2006)]); namely, that there really are two kinds of RG explanations, one given by the real-space (or Kadanoff) approach, the other by a so-called field-theoretic (or Wilsonian) approach.

We have seen above how the real-space approach is supposed to work. And, it is true that physics textbooks often do refer to the field-theoretic approach as distinct. In fact, one text upon which Franklin relies makes the distinction between the two types of RG explanations quite explicit. [Binney et al.(1992)Binney, Dowrick, Fisher, and Newman] I think this distinction (between real-space and field-theoretic RG) makes some sense and does simplify some calculations. However, it begs the important question when it comes to explaining universality. That is, the so-called "field-theoretic" approach assumes universality rather than explaining it.

The field-theoretic "explanation" starts with what is known as the LandauGinzburg-Wilson (LGW) Hamiltonian:

$$
H=\int d^{d} \mathbf{x}\left[\frac{1}{2} \zeta^{2}|\nabla \phi(\mathbf{x})|^{2}+\frac{1}{2} \theta|\phi(\mathbf{x})|^{2}+\frac{1}{4 !} \eta|\phi(\mathbf{x})|^{4}\right] .
$$

For my purposes here it suffices to note two features of this Hamiltonian (neither of which is controversial). First, $\phi(\mathbf{x})$ is an order parameter (like $\Psi$ or $M$ ) and that, within the LGW Hamiltonian, only even powers of that order parameter appear. Second, the LGW Hamiltonian is an effective Hamiltonian. This latter feature means that the LGW Hamiltonian is not a microscopic characterization of the system. In fact, since it makes reference to the order parameter $\phi(\mathbf{x})$, given the discussion in section 3 it is a mesoscale Hamiltonian. Thus, unlike the real-space RG explanation that starts with 
microscopic Hamiltonians, the field-theoretic approach begins with a Hamiltonian that already ignores various microscopic details that distinguish different systems. Most importantly, the LGW Hamiltonian's elimination of odd power terms of the order parameter, is a reflection of the symmetry of that order parameter.

In other words, the LGW Hamiltonian is designed to represent systems that share the same dimensionality and order parameter symmetry. Therefore, it cannot serve to explain why the systems in the universality class have those properties as common features. It is surely true that condensed matter physicists often start with the effective (mesoscopic) LGW Hamiltonian, rather than a more realistic (or at least more detailed), microscopic Hamiltonian. They then use this to understand macroscopic aspects captured, for example, by the power law scaling relations. But what allows them to start at this mesoscopic scale? What, that is, justifies the use of the LGW Hamiltonian as the starting point?

Recall Fisher's remarks in [Fisher(1998)] that Landau's introduction of an order parameter introduced a novel foliation of the world into microscopic, mesoscopic, and macrosopic. The LGW Hamiltonian, lives in the mesoscopic regime. In that same paper, Fisher argues that work needs to be done to justify the use of the LGW Hamiltonian:

... [O]ne starts from this intermediate level with a physically appropriate LGW Hamiltonian in place of a true (or, at least, more faithful or realistic) microscopic Hamiltonian; and then one brings statistical mechanics to bear in order to understand the macroscopic level. The derivation and validity of the many types of initial, LGW Hamiltonians may then be the object of separate studies to relate them to the atomic level." [Fisher(1998), p. 654-655, emphases in original]

These latter, "separate studies" aim to relate the mesoscale to the atomic scale. They include RG investigations of the sort discussed in section 5 . They involve real-space techniques designed to establish the dependence of phase transition problems on dimensionality and symmetry and they work by providing in principle demonstrations that near criticality, the details that genuinely distinguish the systems (and their respective Hamiltonians) from one another are by and large irrelevant for the upper scale behavior of interest. It is only after these demonstrations that one is able to justifiably 
appeal to an effective Hamiltonian as representative of a large (universality) class of distinct systems.

\section{Conclusion}

As Kadanoff and others (specifically Robert Griffiths [Griffiths(1970)]) have emphasized, universal behavior reflects a stability of a certain behavior under perturbation of particular details. The concept of universality also depends upon a separation of scales and the fact that no nearby characteristic length (time) scale is present. Finally, it is part of the very concept of universality of critical phenomena that the (universality) class of systems depends upon the dimensionality of the system and the symmetry of the ordered state. Thus, any explanation for how the universality of critical phenomena can be possible requires demonstrations that the systems are stable under the appropriate $(\lambda$-)perturbation and that the only system features relevant to the behavior are the physical dimensionality and the symmetry of the ordered state.

The renormalization group can provide these two demonstrations. It does so by introducing a transformation on an abstract space of Hamiltonians corresponding to actual and possible systems and finding fixed points of that transformation. The Hamiltonians that flow to the same fixed point are exactly those between which the perturbative $(\lambda)$ stability holds. They are also those critical systems that share dimensionality and the appropriate meso-scale symmetry.

As discussed in section 6 a number of objections have been offered to this RG explanation. I answer these by looking back at the historical discussions in which the concept "universality" first appeared as an expression of similar behavior by radically distinct systems. It seems (to me at least) that the commonality strategy for explaining universality is misguided. In fact, since universality is a statement to the effect that the similar behaviors of critical systems depend only on dimensionality and symmetry, to explain how universal behavior is possible requires explaining the dependence upon dimensionality and symmetry. Simply citing those properties as explanans just won't do the trick. Finally, appealing to a field-theoretic RG that starts with an effective Hamiltonian fails to meet the explanatory task. The effective (LGW) Hamiltonian requires justification. As it is effective, it actually expresses the very universality one seeks to explain. 


\section{References}

[Batterman(2002)] Robert W. Batterman. The Devil in the Details: Asymptotic Reasoning in Explanation, Reduction, and Emergence. Oxford Studies in Philosophy of Science. Oxford University Press, 2002.

[Binney et al.(1992)Binney, Dowrick, Fisher, and Newman] J. J. Binney, N. J. Dowrick, A. J. Fisher, and M. E. J. Newman. The Theory of Critical Phenomena: An Introduction to the Renormalization Group. Oxford University Press, 1992.

[Fisher(1983)] Michael E. Fisher. Scaling, universality and renormalization group theory. In F.J.W. Hahne, editor, Critical Phenomena, volume 186 of Lecture Notes in Physics, Berlin, 1983. Summer School held at the University of Stellenbosch, South Africa; January 18-29, 1982, SpringerVerlag.

[Fisher(1998)] Michael E. Fisher. Renormalization group theory: Its basis and formulation in statistical physics. Reviews of Modern Physics, 70 (2):653-681, 1998.

[Franklin(2017)] Alexander Franklin. On the renormalisation group explanation of universality. Philosophy of Science, 2017.

[Griffiths(1970)] Robert B. Griffiths. Dependence of critical indices on a parameter. Physical Review Letters, 24(26):1479-1482, 1970.

[Guggenheim(1945)] E. A. Guggenheim. The principle of corresponding states. The Journal of Chemical Physics, 13(7):253-261, 1945.

[Kadanoff(1971)] Leo P. Kadanoff. Critical Behavior. Universality and Scaling. In M. S. Green, editor, Proceedings of the International School of Physics "Enrico Fermi" Course LI, volume Course LI, pages 100-117, New York, 1971. Italian Physical Society, Academic Press.

[Kadanoff(1976)] Leo P. Kadanoff. Scaling, universality, and operator algebras. In C. Domb and M. S. Green, editors, Phase Transitions and Critical Phenomena, volume 5A. Academic Press, 1976.

[Kadanoff(2000)] Leo P. Kadanoff. Statistical Physics: Statics, Dynamics, and Renormalization. World Scientific, Singapore, 2000. 
[Kadanoff(2013)] Leo P. Kadanoff. Theories of matter: Infinities and renormalization. In Robert W. Batterman, editor, The Oxford Handbook of Philosophy of Physics, chapter Four, pages 141-188. Oxford University Press, 2013.

[Landau(1965)] L.D̃. Landau. On the theory of phase transistions. In D. Ter Haar, editor, Collected Papers of L. D. Landau, chapter 29, pages 193216. Gordon and Breach, Science Publishers, New York, 1965.

[Lange(2015)] Marc Lange. On "minimal model explanations": A reply to batterman and rice. Philosophy of Science, 82:292-305, 2015.

[Mainwood(2006)] Paul Mainwood. Is More Different? Emergent Properties in Physics. PhD thesis, Merton College, University of Oxford, 2006.

[Reutlinger(2017)] Alexander Reutlinger. Do renormalization group explanations conform to the commonality strategy? Journal of General Philosophy of Science, 48:143-150, 2017. 